\title{
Epidemiology of Hereditary Coagulation Bleeding Disorders: A 15-Year Experience From Southern Iran
}

\author{
Azadeh Farjami', Sezaneh Haghpanah', Peyman Arasteh ${ }^{2,3}$, Rezvan Ardeshiri', Hakimeh Tavoosi ${ }^{1}$, \\ Zohre Zahedi', Shirin Parand', Mehran Karimi ${ }^{1 *}$ \\ ${ }^{1}$ Hematology Research Center, Shiraz University of Medical Sciences, Shiraz, Iran \\ ${ }^{2}$ Noncommunicable Diseases Research Center, Fasa University of Medical Sciences, Fasa, Iran \\ ${ }^{3}$ Department of MPH, Shiraz University of Medical Sciences, Shiraz, Iran
}

*Corresponding Author: Mehran Karimi, M.D., Professor of Pediatric Hematology-Oncology, Hematology Research Center, Shiraz University of Medical Sciences, Shiraz, Iran. Tel: +98-711-6473239,

Email: Karimim@sums.ac.ir

Received September 26, 2017; Accepted October 11, 2017; Online Published October 28, 2017

\begin{abstract}
Background: Data on the frequency of hereditary bleeding disorders (HBDs) and associated mortality and morbidities during a long-term follow-up from Iran are scarce.

Objective: This study evaluated the epidemiologic features among patients with HBD in one of the largest referral centers in southern Iran.

Methods: In this cross-sectional study, 619 patients with HBD were evaluated during the period 1996 to 2011 . Aside from baseline characteristics and type of factor deficiency, associated morbidities including viral infections, neurological disorders, asthma, thalassemia, glucose-6-phosphate dehydrogenase (G6PD) deficiency, diabetes, hypertension, cardiac and renal diseases were evaluated. Furthermore, among patients who died, the underlying disease and etiology of death were also evaluated.

Results: Patients' mean age was $24.4 \pm 13.5$ years. Factor VIII deficiency was the most prevalent type (50.4\%) of HBD, and combined Von-Willebrand and factor XIII deficiency (2.3\%) was the most prevalent type of combined factor deficiency. A total of $0.5 \%$ had hepatitis B and $11.5 \%$ had hepatitis C. Cardiac disease was seen in $1.5 \%$, hypertension in $0.2 \%$, renal disease in $0.2 \%$, and diabetes in $1.3 \%$ of patients. Overall, $5.2 \%$ had intracranial hemorrhage, $2.1 \%$ had epilepsy, and $0.8 \%$ had mental retardation. During the 15-year follow-up, 22 patients died; car accident was the leading cause of death in this population.

Conclusion: Associated morbidities were seen in $24.3 \%$ of patients with HBD. Most prevalent morbidities were HCV infections (11.5\%) and neurological disease (7.3\%). The mortality rate among patients with HBD was $3.4 \%$, and the most common cause of death was accident, which is similar to that of normal Iranian populations.

Keywords: Hereditary Bleeding Disorder, Morbidity, Mortality, Hemophilia
\end{abstract}

\section{Background}

Advances in medical management, especially factor replacement therapy, have led to increased life expectancy and, consequently, a variety of age-related morbidities among patients with hereditary bleeding disorders (HBDs). ${ }^{1}$

This population is at risk of acquiring several viral infections, including $\mathrm{HIV}$, and hepatitis $\mathrm{B}$ virus (HBV) and hepatitis $\mathrm{C}$ virus (HCV) due to frequent blood, fresh frozen plasma (FFP), cryoprecipitate transfusions, and plasma-derived factor replacement therapies. ${ }^{2-4}$

Among associated morbidities seen in this population, $\mathrm{HCV}$ has reportedly been the most common cause of mortality and morbidity, especially before the mid-1980s. ${ }^{3-5}$ Moreover, hepatitis B is now considered the most prevalent liver disease in these patients. ${ }^{6}$ The prevalence rates of $\mathrm{HCV}$ and HBV in Iranian patients with hemophilia have been reported to be $15 \%-80 \%$ and $0.7 \%-27 \%$, respectively. ${ }^{2,3}$ Among other age-related morbidities is ischemic heart disease. Although controversy exists in the literature regarding the prevalence of ischemic heart disease among patients with hemophilia, Lim and Pruthi reported a similar risk of coronary vascular disease in patients with hemophilia compared to a control group. ${ }^{7}$ Hypertension and diabetes are other risk factors for ischemic heart disease with estimated prevalence rates of $65.5 \%$ and $10.3 \%$ in patients with hemophilia, respectively. ${ }^{7}$ Another common morbidity among patients with hemophilia is renal failure. Hematuria is a common symptom in hemophilia, however, its effects on kidney function is unknown. ${ }^{8}$ Ranta

Copyright $\odot 2017$ The Author(s). This is an open-access article distributed under the terms of the Creative Commons Attribution License (http:// creativecommons.org/licenses/by/4.0), which permits unrestricted use, distribution, and reproduction in any medium, provided the original work is properly cited. 
et al found that this population has a higher incidence of chronic renal failure compared to the male population in general. $^{9}$

Patients with HBDs have a higher risk of intracranial hemorrhage $(\mathrm{ICH})$ and its associated complications. In countries with good healthcare systems, the prevalence of ICH during the neonatal period is estimated to be 3.5\%$4 \%$ among male patients, and $\mathrm{ICH}$ after the neonatal period is estimated to be $3 \%-10 \%$ among all patients with hemophilia. ${ }^{10}$

HBDs put a large financial load on the healthcare system. Furthermore, patients with these conditions are exposed to multiple co-morbid diseases, especially with the advances in medical care and increased life expectancy in the past decade. Providing adequate care poses a challenge for both patients and clinicians. Therefore, understanding the epidemiological distribution of the disease and its associated morbidities can help both patients and health policymakers to better plan for these patients. ${ }^{1,3}$

\section{Objective}

In Iran, the prevalence of hemophilia and its associated morbidities have rarely been reported in the settings of a long-term study. In the current study, the frequency, mortality, and morbidities among patients with hereditary coagulation bleeding disorders were studied during a 15year period in one of the largest referral center in southern Iran.

\section{Methods}

\subsection{Setting of the Study}

In this cross-sectional epidemiologic report, patients with HBDs registered at the Fars Hemophilia Center, affiliated with Shiraz University of Medical Sciences, Shiraz, Iran, were evaluated. The Fars Hemophilia Center is located within Dastgheib hospital, which is a tertiary hospital in Shiraz city (the main city in the province). Patients with either suspected or diagnosed bleeding disorders from all medical care centers throughout Fars province and neighboring provinces refer to the center. Upon referral, blood samples are obtained from each patient. Coagulation factors are quantified and a definite diagnosis is set for each individual, after which, patients are given follow-up dates for receiving related treatments and coagulation factor concentrates in the center.

\subsection{Study Design and Protocol}

In this study, a designed data gathering sheet was used to record and review patients' medical records. Individuals diagnosed with any type of bleeding disorder in the center during 1996 to 2011, using a census sampling method, were included in the study. Data was gathered on baseline characteristics, including gender and age, and on disease specifics, including type of disease (based on factor levels), associated morbidities including: HCV, $\mathrm{HBV}$, and HIV infections, cardiac disease, renal disease, diabetes, hypertension and neurological diseases including epilepsy, ICH, brain tumor, and mental retardation. In addition, asthma, thalassemia, and glucose-6-phosphate dehydrogenase (G6PD) deficiency were also evaluated.

Those individuals who had any missing data were contacted and interviewed, and the related data was obtained. Individuals for whom data was missing were excluded from the study.

Furthermore, patients registered with the center who died during the 15-year period were also considered in a separate group for which overall mortality rates, underlying HBD, and etiology of death were registered.

\subsection{Definition of Variables}

Patients were categorized according to age as less than 10 years old, 10-19, 20-29, 30-39, 40-49, 50-59, and over 60 years old for a better description of the population under study.

Types of factor deficiencies were categorized as factors I, V, VII, IX, and XIII, Von-Willebrand disease, Glanzmann's thrombasthenia, fibrinogen deficiency, Bernard-Soulier syndrome, combined factors V and VIII deficiency, and other combined rare deficiencies including factors VII and VIII deficiency, factors VIII and IX deficiency, factors VIII and X deficiency, factor VII deficiency and Von-Willebrand disease, factor VIII deficiency and Von-Willebrand disease, factor IX deficiency and Von-Willebrand disease, and factor $\mathrm{V}$ deficiency and fibrinogen deficiency.

Cardiac disease was classified as either ischemic or congenital heart disease or unknown.

Thalassemia was classified as either minor or major thalassemia.

Among patients who died during follow-up, the etiology of death was classified as suicide, old age, accident, hepatoencephalopathy, drug overdose, respiratory arrest, bleeding, cancer, or myocardial infarction.

\subsection{Statistical Analysis}

Data was analyzed with SPSS ${ }^{\circledR}$ software for windows ${ }^{\circledR}$, version 17, (SPSS Inc., Chicago, IL, USA). Data is presented as frequency and percentage for qualitative variables and means and standard deviations for quantitative variables.

\section{Results}

During the study period, a total of 619 patients with HBDs were registered with the Fars Hemophilia Center. Among them, 497 (77.3\%) were male and 141 (22.7\%) were female.

Mean age of participants was $24.4 \pm 13.5$ (SD) years, and age ranged from 2 to 82 years. Most patients $(72.1 \%)$ were between 10 and 39 years of age, and the most frequent age group was the 20- to 29-year-old group (32.9\%).

Factor VIII deficiency was the most prevalent factor deficiency type (50.4\%). Combined factor deficiency was found in 34 (5.8\%) patients, among which combined VonWillebrand and factor XIII deficiency $(2.3 \%)$ was the most prevalent type (Table 1 ).

Overall, $24.3 \%$ of the population showed morbidities. Hepatitis C infection (11.5\%) and neurological disease 
Epidemiology, Mortality, and Morbidity in Bleeding Disorders

Table 1. Baseline and Clinical Characteristics of Patients ${ }^{\mathrm{a}}$

\begin{tabular}{|c|c|}
\hline Variables & Statistics \\
\hline Age (y) & $24.4 \pm 13.5$ \\
\hline \multicolumn{2}{|l|}{ Gender, No. (\%) } \\
\hline Male & $497(77.3)$ \\
\hline Female & $141(22.7)$ \\
\hline \multicolumn{2}{|l|}{ Age groups - no. (\%) } \\
\hline$<10$ & $100(16.3)$ \\
\hline $10-19$ & $138(22.5)$ \\
\hline $20-29$ & $204(33.2)$ \\
\hline $30-39$ & $105(17.1)$ \\
\hline $40-49$ & $35(5.7)$ \\
\hline $50-59$ & $19(3.1)$ \\
\hline$>60$ & $13(2.1)$ \\
\hline Total & $614(100)$ \\
\hline Factor V, No. (\%) & $5(0.8)$ \\
\hline Factor VII, No. (\%) & $36(5.8)$ \\
\hline Factor IX, No. (\%) & $336(54.4)$ \\
\hline Factor X, No. (\%) & $20(3.2)$ \\
\hline Factor XI, No. (\%) & $10(1.6)$ \\
\hline Factor XIII, No. (\%) & $1(0.2)$ \\
\hline Von-Willebrand disease, No. (\%) & $74(12)$ \\
\hline Glanzmann's thrombasthenia, No. (\%) & $39(6.3)$ \\
\hline Fibrinogen deficiency, No. (\%) & $10(1.6)$ \\
\hline Bernard Soulier syndrome, No. $(\%)$ & $21(3.4)$ \\
\hline Factor V and VIII, No. (\%) & $8(1.3)$ \\
\hline Factor VII and VIII, No. (\%) & $2(0.3)$ \\
\hline Factor VIII and IX, No. (\%) & $4(0.6)$ \\
\hline Factor VIII and X, No. (\%) & $2(0.3)$ \\
\hline Factor VII and Von-Willebrand disease, No. (\%) & $1(0.2)$ \\
\hline Factor VIII and Von-Willebrand disease, No. (\%) & $14(2.3)$ \\
\hline Factor IX and Von-Willebrand disease, No. (\%) & $1(0.2)$ \\
\hline Factor VIII and Glanzmann's thrombasthenia, No. (\%) & $2(0.2)$ \\
\hline Factor $\mathrm{V}$ and fibrinogen deficiency, No. (\%) & $1(0.2)$ \\
\hline Total & $619(100)$ \\
\hline
\end{tabular}

(7.3\%) (seizure and ICH) were the most prevalent morbidities among patients. Five patients had both epilepsy and ICH. Thalassemia was also observed in $2.4 \%$ of patients.

In the population with hemophilia (A and B), from 372 patients, $16.8 \%$ had hepatitis $\mathrm{C}, 0.5 \%$ had hepatitis $\mathrm{B}$, and $6.2 \%$ had neurological disease (Table 2).

Between the years of 1996 and 2011, 22 patients with HBDs died (3.4\%). From these patients, $50 \%$ were female and $50 \%$ were male. The most frequent factor deficiency among this group was factor VIII deficiency (45.4\%). The most common cause of death was car accident (22.7\%), followed by unrelated or unknown causes (like old age, etc) (18.1\%) and bleeding (18.1\%); 2 patients died from severe epistaxis and 2 died from ICH (Table 3 ).

\section{Discussion}

This study determined the epidemiologic features and
Table 2. Associated Diseases Among Patients With Bleeding Tendencies ${ }^{\mathrm{a}}$

\begin{tabular}{|c|c|c|}
\hline Variables & Subgroups & Frequency \\
\hline Hepatitis B & & $3(2)$ \\
\hline Hepatitis C & & $71(47)$ \\
\hline HIV & & 0 \\
\hline \multirow[t]{4}{*}{ Cardiac disease } & & $9(6)$ \\
\hline & Ischemic disease & 1 \\
\hline & Congenital disease & 3 \\
\hline & Unknown & 5 \\
\hline Hypertension & & $1(0.6)$ \\
\hline Renal disease & & $1(0.6)$ \\
\hline Mental retardation & & $5(3)$ \\
\hline Diabetes & & $8(5)$ \\
\hline Brain mass & & 0 \\
\hline \multirow[t]{3}{*}{ Neurological disease } & & $45(30)$ \\
\hline & Intracranial hemorrhage & 32 \\
\hline & Epilepsy & 13 \\
\hline \multirow[t]{3}{*}{ Thalassemia } & & $14(9)$ \\
\hline & Minor & 13 \\
\hline & Major & 1 \\
\hline G6PD deficiency & & $3(2)$ \\
\hline Asthma & & $3(2)$ \\
\hline Total & & $150(24.3)$ \\
\hline
\end{tabular}

Abbreviation: G6PD, glucose-6-phosphate dehydrogenase deficiency.

${ }^{a}$ All percentages are compared to the overall population with morbidity.

associated morbidity and mortality among patients with bleeding disorders during a 15-year period in one of the largest hemophilia centers in Iran. To the best of the authors' knowledge, this is among the first reports from Iran on HBDs with a long follow-up period.

In the current study, the prevalence rates of hepatitis $\mathrm{B}$ and $\mathrm{C}$ were $0.5 \%$ and $11.5 \%$, respectively, among 619 patients with heredity bleeding disorders. Among the 372 patients with hemophilia, the prevalence rates of hepatitis B and $\mathrm{C}$ were $0.5 \%$ and $16.8 \%$, respectively. Different reports exist from different parts of Iran regarding associated diseases in hemophilia. The prevalence of hepatitis C among patients with hemophilia A and B in Isfahan was reported to be $66 \% .^{3}$ In a study from southeastern Iran, the prevalence rates of hepatitis $\mathrm{B}$ and $\mathrm{C}$ in patients with hemophilia were $4.9 \%$ and $29.6 \%,{ }^{11}$ respectively, and in Ahvaz city, in southwestern Iran, the prevalence rates were $1.1 \%$ and $54 \%,{ }^{3}$ respectively. Overall, the prevalence rates of $\mathrm{HCV}$ and $\mathrm{HBV}$ in Iranian hemophiliac patients are reported to be $15 \%-80 \%$ and $0.7 \%-27 \%$, respectively. ${ }^{2,3,12}$

One reason for the lower rates of $\mathrm{HBV}$ and $\mathrm{HCV}$ in this study may be due to the higher availability of factor concentrates for transfusion, instead of whole blood transfusion, compared to other centers in the country, as the study center is a major referral center in southern Iran.

None of the patients in this study had HIV; however, in a study by Alavian et al among hemophilic patients referring to the Iran Hemophilic Society, the prevalence of HIV was reported to be $2.3 \%{ }^{13}$

The prevalence rates of diabetes and hypertension 
Table 3. Mortality-Related Features Among Patients ${ }^{\mathrm{a}}$

\begin{tabular}{lc}
\hline Variables & Frequency \\
\hline Overall mortality & $22(100)$ \\
Underlying disease among patients who died & \\
Factor VIII & $10(45.4)$ \\
Von-Willebrand disease & $4(18.1)$ \\
Factor XI & $1(4.5)$ \\
Factor V & $1(4.5)$ \\
Factor VII & $1(4.5)$ \\
Glanzmann's thrombasthenia & $1(4.5)$ \\
Factor VIII and Von-Willebrand disease & $1(4.5)$ \\
Factor IX & $2(9.5)$ \\
Factor 1 & $1(4.5)$ \\
Etiology of mortality among patients & \\
Suicide & $1(4.5)$ \\
Unrelated or unknown & $4(18.1)$ \\
Accident & $5(22.7)$ \\
Hepatoencephalopathy & $1(4.5)$ \\
Drug overdose & $3(13.6)$ \\
Respiratory arrest & $1(4.5)$ \\
Bleeding & $4(18.1)$ \\
Cancer & $1(4.5)$ \\
Myocardial infarction & $2(9)$ \\
\hline
\end{tabular}

a Overall, 22 individuals from a total of 641 registered patients (3.4\%) died during the study period.

as risk factors for coronary vascular disease were also determined. Among the 619 patients, 8 individuals had diabetes (1.3\%) and 1 patient had hypertension (0.2\%). Khleif et al determined the prevalence of hypertension to be $46 \%$ and the prevalence of diabetes to be $14 \%$ in their study of individuals with hemophilia. This difference in prevalence rates could be attributed to multiple factors, like different lifestyles and genetic backgrounds. Moreover, unlike the current study in which the majority of patients were below the age of 40 years, Khleif et al included middle-aged and elderly patients in their study. ${ }^{1}$ Fransen van de Putte et al studied hemophilia patients aged over 30 years and found the prevalence of hypertension to be $49 \% .^{14}$ In their study of a US population aged more than 35 years old with hemophilia, Lim and Pruthi found that the prevalence rates of hypertension and diabetes were $65.5 \%$ and $10.3 \%$, respectively. ${ }^{7}$ The prevalence rates of diabetes and hypertension in the current study $(1.3 \%$ and $0.2 \%$, respectively) were less than that reported in other studies, which may be due to the younger population of study (mostly below 40 years of age).

Regarding hematuria and its effects on the kidney which is still unknown, ${ }^{15}$ among the patients in this study with HBDs, 11 (1.8\%) had periodic hematuria; only 1 patient (0.2), however, had chronic renal disease and required dialysis. Khleif et al determined the prevalence of chronic renal disease to be $8 \%$ in their study. ${ }^{1}$ Renal function in patients of the current study was not determined using a specific test, and this needs further evaluation.
The prevalence of ICH was 5.2\%. In their study, Klinge et al found the prevalence of ICH among patients with hemophilia was $4 \%,{ }^{16}$ which was similar to that of the current study. In addition, ICH has also been reported to be higher than the normal population in patients with factor X, factor V, factor VII, and factor XIII deficiencies. ${ }^{17}$

Khleif et al found the prevalence of neurological diseases including seizure, Parkinson disease, and dementia to be $5 \%$. They also found the prevalence of respiratory diseases, including asthma, to be $11 \%$ among these patients. ${ }^{1}$ In the current study population, however, a lower prevalence of asthma $(0.5 \%)$ was found. This could be, as mentioned before, related to the differences in the baseline characteristics of the populations in the two studies as Khleif et al included older patients who would naturally have more comorbidities.

In the current study, 4 patients died due to bleeding and 1 patient died due to complications associated with hepatitis C. In his study among Dutch hemophilia patients, Triemstra et al found a mortality rate of $5 \%$; one patient died from ischemic heart disease, which was similar to the current findings ( 2 deaths occurred due to myocardial infarction in the current study).$^{18}$ In his study among Italian hemophilia patients, Tagliaferri et al determined the mortality rate to be $1.08 \%$ between the years of 2000 and $2007 .{ }^{19}$

The current study was not without limitation. Although it did have one of the longest follow-ups in the literature, the retrospective nature of the study did not allow a complete evaluation of patients' current disease status. However, interviews were performed to make up for any missing data. Although the study was conducted in one of the largest hemophilia centers in the country (the largest center in southern Iran), it only included a single center design and may not be representative of the whole country.

Multi-center studies are needed to identify morbidities associated with bleeding disorders and to choose the best management protocol for improving quality and the health status of patients with bleeding disorders.

\section{Conclusion}

Based on the current results, the prevalence rate of morbidities in patients with HBDs was $24.3 \%$. The most prevalent morbidities were $\mathrm{HCV}$ infection (11.5\%) and neurological disease $(7.3 \%)$. The mortality rate among patients with HBDs was $3.4 \%$, and the most common cause of death was accidents, which is similar to that of the normal Iranian population.

\section{Authors' Contributions}

$\mathrm{MK}$ and $\mathrm{SH}$ aided in the development of the study concept. $\mathrm{MK}, \mathrm{SH}$, and AF aided in study development. RA, HT, $\mathrm{ZZ}$, and SP participated in data acquisition. $\mathrm{AF}, \mathrm{SH}$, and $\mathrm{PA}$ aided in interpretation of data. $\mathrm{SH}$ and $\mathrm{PA}$ aided in statistical analysis. AF and PA aided in preparation of the manuscript. SH, MK, RA, HT, ZZ, and SP aided in a significant revision of the final manuscript. 


\section{Research Highlights}

\section{What Is Already Known?}

Frequencies of HBDs and their associated morbidities are well known and documented in the Western world when compared to that of the middle-east.

\section{What This Study Adds?}

The results of this study can be considered a novel representation of the characteristics of HBDs in southern Iran during a long-term follow-up.

\section{Conflict of Interest Disclosures}

Authors have no conflicts of interest to declare regarding any part of the manuscript.

\section{Ethical Approval}

Informed and written consent was obtained from all participants and their parents in cases of underaged children. The study protocol and design was approved by the Institutional Review Board (IRB) of Shiraz University of Medical Sciences (Ethics code \#2969).

\section{Acknowledgments}

The authors would like to thank all patients and their families for taking part in the study. We would also like to thank all personnel at the Shiraz Hemophilia Center who aided in the acquisition of data.

\section{References}

1. Khleif AA, Rodriguez N, Brown D, Escobar MA. Multiple comorbid conditions among middle-aged and elderly hemophilia patients: prevalence estimates and implications for future care. J Aging Res. 2011;2011:985703. doi:10.4061/2011/985703

2. Assarehzadegan MA, Ghafourian Boroujerdnia M, Zandian K. Prevalence of hepatitis B and C infections and HCV genotypes among haemophilia patients in haves, southwest Iran. Iran Red Crescent Med J. 2012;14(8):470-474.

3. Yazdani MR, Kassaian N, Ataei B, Nokhodian Z, Adibi P. Hepatitis $C$ virus infection in patients with hemophilia in Isfahan, Iran. Int J Prev Med. 2012;3(suppl 1):S89-93.

4. Zhang M, Rosenberg PS, Brown DL, et al. Correlates of spontaneous clearance of hepatitis C virus among people with hemophilia. Blood. 2006;107(3):892-897. doi:10.1182/ blood-2005-07-2781.

5. Gadalla SM, Preiss LR, Eyster ME, Goedert IJ. Correlates of high hepatitis C virus RNA load in a cohort of HIV-negative and HIV-positive individuals with haemophilia. J Viral Hepat.
2011;18(3):161-169. doi:10.1111/j.1365-2893.2010.01289.x.

6. Arababadi MK, Nasiri Ahmadabadi B, Yousefi Daredor H, Kennedy D. Epidemiology of occult hepatitis B infection among thalassemic, hemophilia, and hemodialysis patients. Hepat Mon. 2012;12(5):315-319. doi:10.5812/hepatmon.5934.

7. Lim MY, Pruthi RK. Cardiovascular disease risk factors: prevalence and management in adult hemophilia patients. Blood Coagul Fibrinolysis. 2011;22(5):402-406. doi:10.1097/ MBC.0b013e328345f582.

8. Kulkarni R, Soucie JM, Evatt BL. Prevalence and risk factors for heart disease among males with hemophilia. Am J Hematol. 2005;79(1):36-42. doi:10.1002/ajh.20339.

9. Ranta S, Valta H, Viljakainen $\mathrm{H}$, Makitie $\mathrm{O}$, Makipernaa A. Hypercalciuria and kidney function in children with haemophilia. Haemophilia. 2013;19(2):200-205. doi:10.1111/ hae. 12021

10. Ljung RC. Intracranial haemorrhage in haemophilia A and B. Br J Haematol. 2008;140(4):378-384. doi:10.1111/j.13652141.2007.06949.x.

11. Sharifi-Mood B, Eshghi P, Sanei-Moghaddam E, Hashemi M Hepatitis B and C virus infections in patients with hemophilia in Zahedan, southeast Iran. Saudi Med J. 2007;28(10):15161519.

12. Karimi M, Hashemi A, Ghiam AF, Jahromi SS, Toobaee S. Substance dependency in Iranian patients with hemophilia. Addict Behav. 2007;32(2):365-369.doi:10.1016/j.addbeh.2006.03.040.

13. Alavian SM, Ardeshiri A, Hajarizadeh B. Prevalence of HCV, HBV and HIV infection among hemophilia patients. Hakim Res J. 2003;6(2):45-52. [Persian].

14. Fransen van de Putte DE, Fischer $K$, Makris $M$, et al. Unfavourable cardiovascular disease risk profiles in a cohort of Dutch and British haemophilia patients. Thromb Haemost. 2013;109(1):16-23. doi:10.1160/th12-05-0332.

15. Kulkarni R, Soucie JM, Evatt B. Renal disease among males with haemophilia. Haemophilia. 2003;9(6):703-710. doi:10.1046/ j.1351-8216.2003.00821.x.

16. Klinge J, Auberger K, Auerswald G, Brackmann HH, MauzKorholz C, Kreuz W. Prevalence and outcome of intracranial haemorrhage in haemophiliacs--a survey of the paediatric group of the German Society of Thrombosis and Haemostasis (GTH). Eur J Pediatr. 1999;158 Suppl 3:S162-165. doi:10.1007/ PL00014346.

17. Naderi M, Zarei T, Haghpanah S, Eshghi P, Miri-Moghaddam E, Karimi M. Intracranial hemorrhage pattern in the patients with factor XIII deficiency. Ann Hematol. 2014;93(4):693-697. doi:10.1007/s00277-013-1918-7.

18. Triemstra M, Rosendaal FR, Smit C, Van der Ploeg HM, Briet E. Mortality in patients with hemophilia. Changes in a Dutch population from 1986 to 1992 and 1973 to 1986 . Ann Intern Med. 1995;123(11):823-827. doi:10.7326/0003-4819-12311-199512010-00002.

19. Tagliaferri A, Rivolta GF, lorio A, et al. Mortality and causes of death in Italian persons with haemophilia, 1990-2007. Haemophilia. 2010;16(3):437-446. doi:10.1111/j.13652516.2009.02188.x. 